\title{
Evaluation of Method of Measuring Distance Between Object and Walls Using Ultrasonic Sensors
}

\author{
Manabu Ishihara ${ }^{1}$, Makoto Shiina ${ }^{2}$, and Shin-nosuke Suzuki ${ }^{3}$ \\ ${ }^{I}$ Department of Electrical and Computer Engineering, Oyama National College of Technology, ishihara@oyama-ct.ac.jp \\ ${ }^{2}$ Advance Course of Electrical and Computer Engineering, Oyama National College of Technology \\ ${ }^{3}$ Department of Electrical and Computer Engineering, Oyama National College of Technology, shin-s@oyama-ct.ac.jp
}

\begin{abstract}
This paper is concerned with the development of an ultrasonic wave sensor system. A development of the sensor system a the robot which moves in the parallel to the wall is proposed. Ultrasonic wave frequency is $40 \mathrm{kHz}$. The sensor output is calculated with a PIC (computer), and it is displayed by a LCD screen. The architecture to introduce an ultrasonic wave sensor system for the moving environment is proposed. This architecture is simple, so it is applicable to measurement of the positioning control study. A sensor system was developed using only inexpensive and easily available components. Based on the information obtained while designing, manufacturing and testing the prototype, it was concluded that the system proved to be sufficiently capable for use by robots.
\end{abstract}

\section{Keywords}

ultrasonic sensors, PIC, anticollision, wall trace system, small traveling robot

\section{INTRODUCTION}

Ultrasonic sensors are used in various fields because of their unique characteristics. One such use is to provide positioning control for robots. Although ultrasonic ranging provides excellent distance resolution, one of its shortcomings is that it provides low directionality when compared to optical sensors. Various attempts have been made to improve measurement resolution, including the use of rotating and/or sensors capable of operating on multiple frequencies, as well as the adoption of sophisticated signal processing techniques [Okugumo et al., 2008; Nagashima et al., 1994].

In this study, a method believed to be capable of controlling a moving body in a way that allows it to maintain a user-specified distance from nearby walls was investigaed. The method uses multiple ultrasonic sensors and a simple signal processing method.

\section{SENSOR OUTLINE}

\subsection{Ultrasonic sensor characteristics}

One of the characteristics of ultrasonic sensors is that their aerial transmission speed is slower than light, which makes signal processing easier. Furthermore, ultrasonic wavelengths are relatively short (approximately $8.6 \mathrm{~mm}$ at $40 \mathrm{kHz}$ ), which allows for high resolution in the direction of the linear distance measurement. This makes it possible to conduct highly accurate distance measurements. Another advantage is that ultrasound is unaffected by the color of an object and can thus be used to measure the distance from a sensor to a transparent body such as a glass object. Ultrasound is also relatively immune to effects of light and airborne dust, which makes it useful for performing measurements in outdoor environments. Because of these characteristics, ultrasonic sensors are also used for automobile navigation and ranging systems, etc.

\subsection{Conventional sensor system}

One example of a conventional ultrasonic sensor system is an ultrasonic rangefinder. The ultrasonic rangefinder radiates a burst pulse of ultrasound. It measures distances by calculating the reflex-time of the ultrasound reflection from the target-object [Shiina et al., 2008; Ishihara et al., 2008].

Figure 1 shows this system. Ultrasonic sensors are generally used for anticollision purposes by measuring the distance to an obstacle.

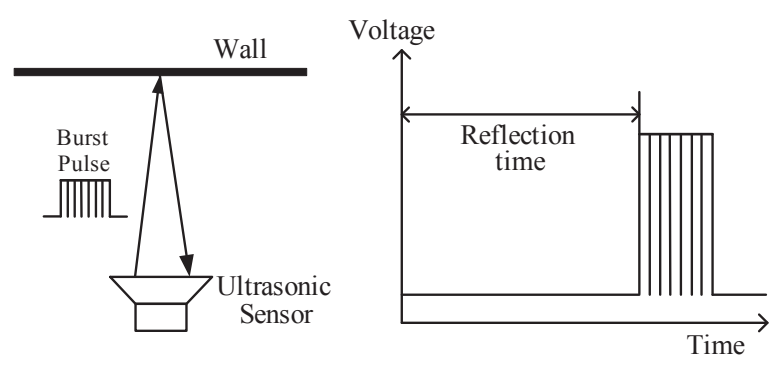

Fig. 1 Ultrasonic measurement system

While it is possible to use multiple units for this type of echo-ranging, as shown in Figure 2, adequate distance between the sensors as well as careful consideration of the arrangement of multiple sensors is required 


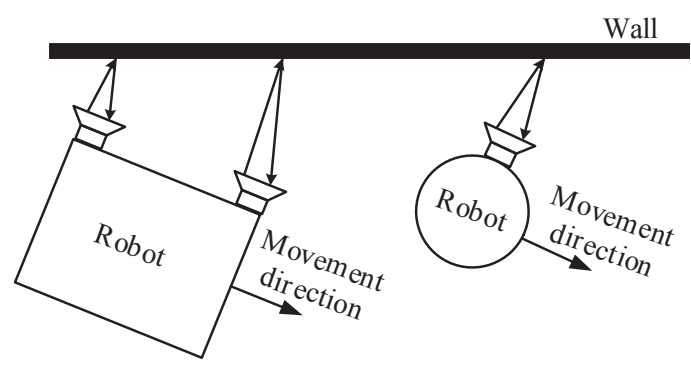

Fig. 2 A sensor arrangement idea

in situations where the robot is cylindrically shaped.

\section{PROPOSED SYSTEM}

\subsection{Measurement principle}

A situation where a robot is required to move parallel to a wall was examined, as shown in Figure 3. If it is possbile to somehow simultaneously measure the minimum distance $\mathrm{x}$, between the system and the wall, and the distance to the wall $\mathrm{y}$, in the frontal direction of the system, (as seen in Figure 4) it is known that the robot is moving parallel to the wall when $\mathrm{x}$ and $y$ are equal. Using these measurements, the system's inclination angle can also be determined.

The relation between the angle $\theta, \mathrm{x}$ and $\mathrm{y}$ is expressed as:

Equation

$$
\cos \theta=\frac{x}{y}
$$

Then,

Equation

$\theta=\cos ^{-1} \frac{x}{y}$

Thus, the system is controlled to satisfy the relation $\theta$ $=0$ [deg] in the equation (2). When a robot is controlled in a real system, the $\mathrm{x}$ and $\mathrm{y}$ values are measured constantly and the robot's movements are controlled to ensure they stay within the given threshold values. In addition, because this method uses measured angles as parameters, positioning control can be determined easily.

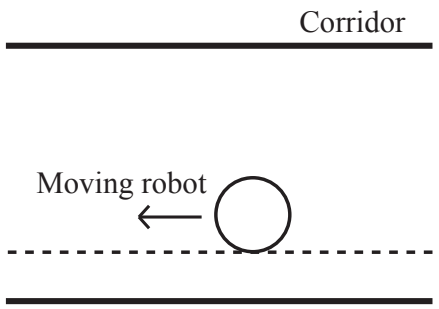

Fig. 3 Equidistant control

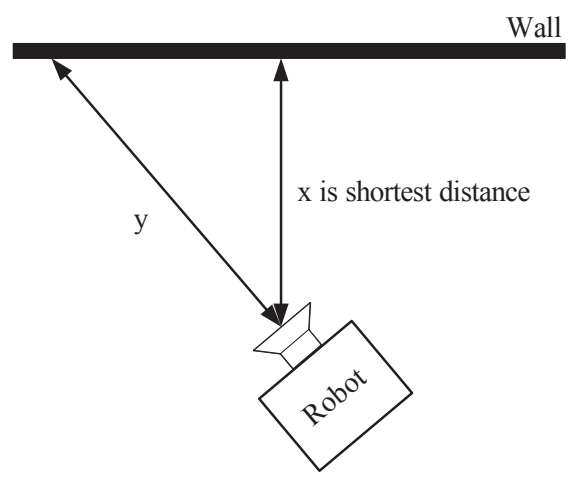

Fig. 4 Measurement system outline

\subsection{Minimum distance measurement}

The minimum distance between the wall and the system $\mathrm{x}$ has to be measured even when the system (the axis of the sensor) is somewhat tilted, as described above. Here, the sensor for measuring the distance $\mathrm{x}$ is called X. When measuring the minimum distance, the broad directional angle of the ultrasonic sensor is utilized. As shown in Figure 5 (a), ultrasonic sensors typically have a wide measurable range, i.e. directional angle. In other words, even when the ultrasonic sensor is slightly tilted, as shown in Figure 5 (b), the measured distance is approximately equal to the minimum distance $\mathrm{x}$. This system measures the ambspace (the minimum distance) using this feature.

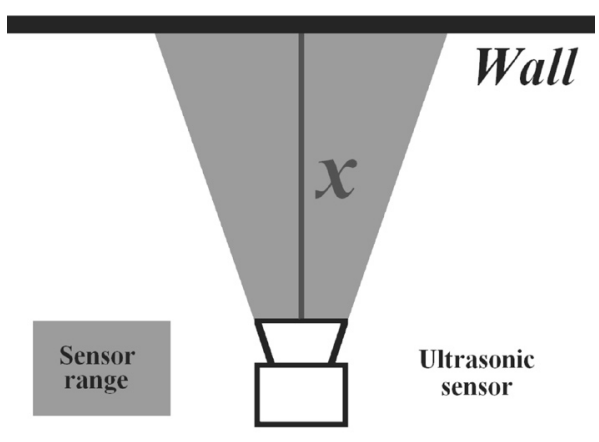

(a) Perpendicular to a wall

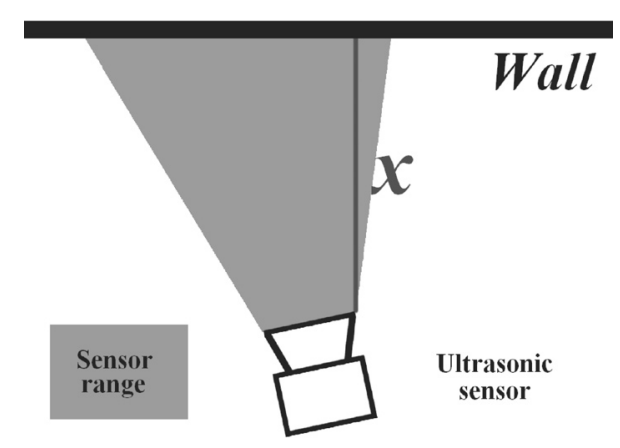

(b) Direction change

Fig. 5 Shortest distance measurement by the ultrasonic sensor 


\subsection{Tilted distance measurement}

If the system (the axis of the sensor) is not precisely perpendicular to the wall, $x \neq y$, it is necessary to measure the tilted distance to the wall y. The sensor for measuring the distance $y$ is called $\mathrm{Y}$, which is shown in Figure 6. In this situation, the distance $y$ is measured from the outermost part of the measuring range of sensor Y. An experiment was therefore conducted to determine the directionality of the sensor.

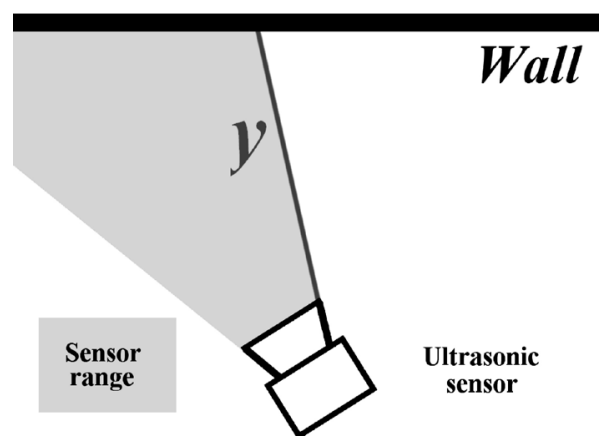

Fig. 6 Changing the rotation direction using the ultrasonic sensor

\subsection{Measurement of minimum distance from wall and tilt angle}

Figure 7 is a conceptual drawing of the system used to simultaneously measure the minimum distance of the system from the wall and the tilt angle $\theta$. In this configuration, the minimum distance $\mathrm{x}$ can be measured with sensor $X$, and the tilt angle of the system can be measured with sensor Y. If the system is tilted in the opposite direction, sensors $\mathrm{X}$ and $\mathrm{Y}$ reverse roles. By simultaneously measuring the minimum distance and the system's tilt, the distance between the robot and the wall can be measured and it can be determined whether the robot is moving parallel to the wall.

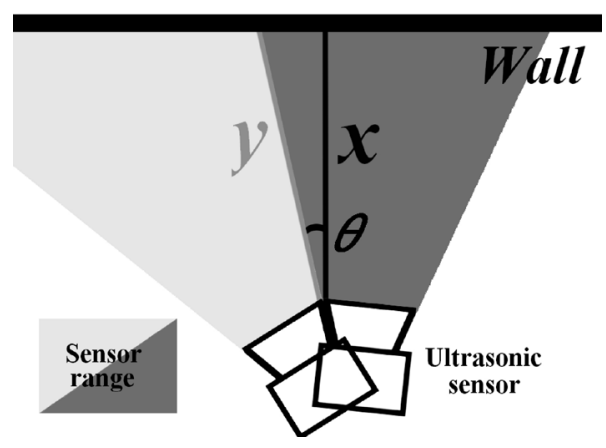

Fig. 7 Angle of tilt and shortest distance measurement

\subsection{System configuration and specification}

An external view of the system is shown in Figure 8. The system uses a microcontroller PIC16F877A (Microchip Technology Inc.) as its primary computer for controlling sensors and calculating tilt angle. It also

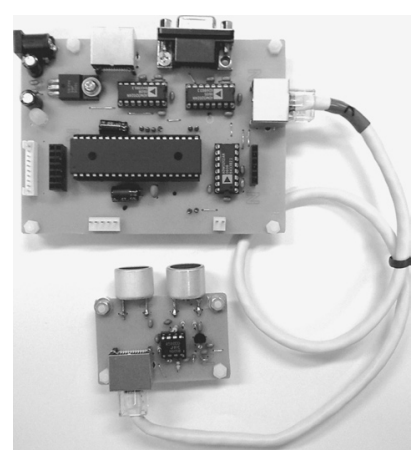

(a) Entire system

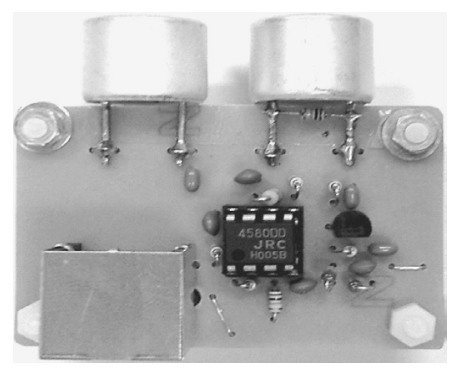

(b) Sensor board

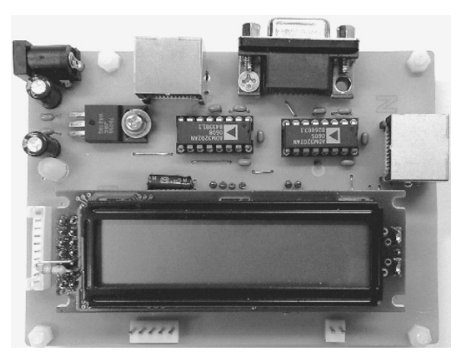

(c) Main board

Fig. 8 System model

uses an Aerial Ultrasonic Sensors T40-16A1 (emitter) and a R40-16A1 (receiver) (Nippon Ceramic Co., Ltd).

The burst pulse for driving the ultrasonic sensor is generated by the PIC microcontroller, and amplified by ADM3202, an RS-232 driver IC from Analog Devices, Inc., before it is output to the ultrasonic sensor. A charged pump type booster circuit is built into ADM3202. It boosts the burst pulse output by the PIC from $5 \mathrm{Vp}$-p to $20 \mathrm{Vp}-\mathrm{p}$.

The sensor board shown in Figure 8 (b) is connected to the main board shown in Figure 8 (c) by means of a LAN cable.

The measured distance and the calculated tilt angle are displayed on a 16-character 2-line character liquid crystal module, SC1602BS (Sunlike Display Tech Corp., Taiwan), which is installed on the main board. Data can also be displayed on a 20-character 4-line character liquid crystal module, SC2004CS (Sunlike Display Tech Corp., Taiwan).

For use as external I/O terminals, a surface-mounted RS-232 port is available and can be used to provide 
communication with the PC (PC-TA compatible machine). Furthermore, an $\mathrm{I}^{2} \mathrm{C}$ port is similarly provided for communication with the PIC microcontroller (PIC16F877A (Microchip Technology Inc., Arizona)).

\section{EXPERIMENT}

\subsection{Distance measurement using ultrasonic sensors}

The sensor's responsiveness was measured by setting it up so that its axis was perpendicular to a wall, and then varying its distance from the wall. The shortest detection distance utilized in this experiment was 33 $\mathrm{cm}$ in order to prevent any test errors, which might otherwise result if the ultrasonic wave curves around a phenomenon. Measurements were then taken for the distances from 50 to $250 \mathrm{~cm}$.

Figure 9 shows the test results. The broken line represents the actual distance, while the measured distances are shown by diamond-shaped dots. The test results show that a linear relation exists between them. The measured data errors fell within a range of $3 \%$, and

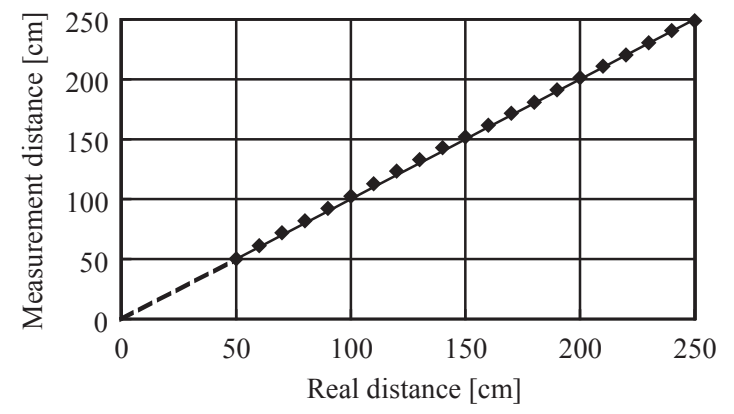

the test proved that the measurement is stable up to $250 \mathrm{~cm}$.

\subsection{Distance measurement using ultrasonic sensors}

Ultrasonic sensor directionality was measured by varying the distance of the ultrasonic sensor from the wall at increments of $25 \mathrm{~cm}$ within a range of 50-150 $\mathrm{cm}$, and then measuring the directional angle at each position by varying the sensor angle. The directional angle is defined as an angle at which the measured distance is judged to coincide with the distance $\mathrm{x}$. The results are shown in Table 1.

It can be seen that the directional angle decreases as the distance increases. This finding is consistent with the directionality of conventional ultrasonic sensors.

Table 1 Directivity Experiment

\begin{tabular}{|l|c|c|c|c|c|}
\hline $\begin{array}{l}\text { Distance with } \\
\text { a wall }[\mathrm{cm}]\end{array}$ & 50 & 75 & 100 & 125 & 150 \\
\hline $\begin{array}{l}\text { Angle of } \\
\text { inclination [deg] }\end{array}$ & 22 & 18 & 15 & 14 & 15 \\
\hline
\end{tabular}

\subsection{Measurement of proposed system characteristics}

The proposed system's characteristics were measured. The angle $\theta$ between the directions of sensor $\mathrm{X}$ and sensor $\mathrm{Y}$ was determined from the results of the second experiment, and a $\theta$ of approximately 20 [deg] was used. The mean and the standard deviation results calculated for each location after four measurements are shown in Table 2 and Figure 10.

Fig. 9 Linear characteristic of proposed system

Table 2 System experiment

(a) Measurement distance

\begin{tabular}{|c|c|c|c|c|}
\hline \multirow{2}{*}{ Angle $[\mathrm{deg}]$} & \multicolumn{2}{|c|}{ Sensor X } & \multicolumn{2}{c|}{ Sensor Y } \\
\cline { 2 - 5 } & Mean x [cm] & Standard deviation x [cm] & Mean y [cm] & Standard deviation y [cm] \\
\hline 0 & 51.7 & 0 & 51.4 & 0.1 \\
\hline 5 & 51.7 & 0.1 & 52.8 & 0.3 \\
\hline 10 & 51.7 & 0 & 53.5 & 0.2 \\
\hline 15 & 51.7 & 0 & 55.3 & 0.1 \\
\hline 17 & 51.9 & 0 & 57.3 & 0.9 \\
\hline 20 & 52.4 & 0 & $\infty$ & - \\
\hline
\end{tabular}

(b) Measurement angle

\begin{tabular}{|c|c|c|}
\hline \multirow{2}{*}{ Angle $[\mathrm{deg}]$} & Mean $\theta[\mathrm{deg}]$ & Measurement angle \\
\cline { 2 - 3 } & 0 & standard deviation $\theta[\mathrm{deg}]$ \\
\hline 0 & 4.0 & 2.52 \\
\hline 5 & 9.6 & 1.36 \\
\hline 10 & 17.9 & 0.42 \\
\hline 15 & 22.6 & 2.14 \\
\hline 17 & 90 & - \\
\hline 20 & & \\
\hline
\end{tabular}




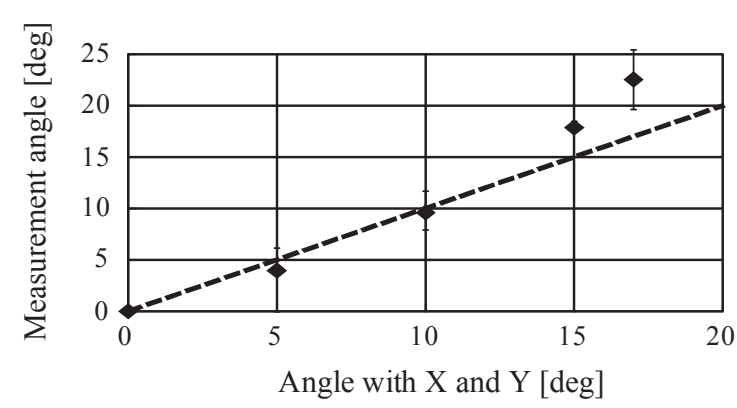

Fig. 10 Angle characteristic of proposed system

\section{DISCUSSION}

Using the proposed sensor system, the distance between the sensor and the wall and the angle of the sensor relative to the wall were measured at distances of 50-250 cm and at angles of 0-15 degrees ( 0 degree indicates that the sensor axis is perpendicular to the wall). From these results, it was concluded that the proposed system is suitable for use as the positioning control of a robot that is designed to travel along a passage, such as a corridor.

Since the proposed system can be connected to the position control circuit of a robot using other microcontrollers via $\mathrm{I}^{2} \mathrm{C}$, or to a PC via RS-232, it can be easily installed and used in other systems as a positiondetecting module.

From these observations, it can be said that the proposed sensor system offers sufficient functionality to allow its use as a position controlling platform for a robot, and that it can be applied to small traveling robots.

\section{CONCLUSION}

A sensor system was developed using only inexpensive and easily available components. Based on the information obtained while designing, manufacturing and testing the prototype, it can be concluded that the system proved to be sufficiently capable for use in robots. Future research will include achieving positioning control of a small robot using the present system.

\section{References}

Okugumo, M., A. Kimura, M. Ohki, and M. Ohkita, Development research on high performance ultrasound sensor system, IEEJ Transactions on Electronics, Information and Systems, Vol. 128, No. 1, 55-61, 2008.

Nagashima, Y., A. Ohya, and S. Yuta, Ultrasonic sensor for mobile robot to measure the normal direction of walls, The Journal of the Acoustical Society of Japan, Vol. 51, No. 1, 39-42, 1994.

Shiina, M. and M. Ishihara, A Positioning Measurement using Ultrasonic Sensor, Proceedings of
ROBOMEC 2008, 1A1-I06 (1-3), 2008.

Ishihara, M., M. Shiina, and S. Suzuki, Development of ultrasonic sensor for mobile robot to measure the normal direction and parallel of walls, Proceedings of Ultrasonic Electronics 2008, 1P10-3, 157-158, 2008.

(Received December 16, 2008; accepted February 9, 2009) 